\title{
Minimal surfaces and symplectic structures of moduli spaces
}

\author{
Brice Loustau
}

Given a closed surface $S$ of genus at least 2, we compare the symplectic structure of Taubes' moduli space of minimal hyperbolic germs with the Goldman symplectic structure on the character variety $\mathcal{X}(S, P S L(2, \mathbb{C}))$ and the affine cotangent symplectic structure on the space of complex projective structures $\mathcal{C P}(S)$ given by the Schwarzian parametrization. This is done in restriction to the moduli space of almost-Fuchsian structures by involving a notion of renormalized volume, used to relate the geometry of a minimal surface in a hyperbolic 3-manifold to the geometry of its ideal conformal boundary.

\section{Contents}

1 Introduction

2 Complex projective structures and symplectic structures 4

$2.1 \mathcal{C P}(S)$ and $\mathcal{T}(S) \ldots \ldots \ldots \ldots \ldots$

2.2 Fuchsian and quasi-Fuchsian projective structures . . . . . . . . 5

2.3 The character variety . . . . . . . . . . . . . . 6

2.4 Schwarzian parametrization of $\mathcal{C P}(S)$ and the affine cotangent symplectic structures .................... 8

3 Minimal surfaces in hyperbolic 3-manifolds, Taubes' moduli space and almost-Fuchsian structures $\quad 10$

3.1 Minimal surfaces in hyperbolic 3-manifolds . . . . . . . . . . . . . 10

3.2 Taubes' moduli space . . . . . . . . . . . . . . . . . . 11

3.3 Almost-Fuchsian structures . . . . . . . . . . . . . . . 12

4 Renormalized volume of almost-Fuchsian manifolds 13

4.1 Equidistant foliations in quasi-Fuchsian 3-manifolds . . . . . . . . . 13

4.2 Definition of the renormalized volume . . . . . . . . . . . 14

4.3 Variation of the renormalized volume, comparing symplectic structures 14 


\section{Introduction}

Let $S$ be a closed oriented surface of genus $g \geqslant 2$. Several moduli spaces associated to $S$ are known to enjoy natural real or complex symplectic structures. Let us mention here those of interest for the purpose of this paper, more precise definitions will follow in the subsequent sections.

- The Teichmüller space of $S$ denoted by $\mathcal{T}(S)$ is the deformation space of complex structures on $S$. It is equipped with a symplectic structure $\omega_{W P}$ which is the Kähler form of the so-called Weil-Petersson metric. In various ways, $\mathcal{T}(S)$ will be seen as a half-dimension "slice" of all the following deformation spaces.

- The character variety $\mathcal{X}(S, G)$ where $G$ is a reductive Lie group is a quotient of the space of group homomorphisms $\operatorname{Hom}\left(\pi_{1}(S), G\right)$. Following Atiyah-Bott AB83, Goldman Gol84 showed that it enjoys a natural symplectic structure $\omega_{G}$ that generalizes the Weil-Petersson symplectic structure on Teichmüller space $(\mathcal{T}(S)$ can be identified as a subspace of the character variety when $P S L(2, \mathbb{R}) \hookrightarrow G$ by the uniformization theorem). When $G$ is a complex Lie group, $\omega_{G}$ is a complex symplectic form. We will be mostly interested in the case $G=P S L(2, \mathbb{C})$.

- Taubes' moduli space $\mathcal{H}$ of minimal hyperbolic germs (introduced by Taubes in [Tau04]) consists of pairs $(I, I I)$ of a first and second fundamental form on $S \hookrightarrow S \times \mathbb{R}$ for the germ of a hyperbolic metric on $S \times \mathbb{R}$ such that $S$ is minimally embedded. $\mathcal{H}$ comes naturally equipped with a real symplectic structure $\omega_{\mathcal{H}}$.

- The deformation space $\mathcal{C P}(S)$ of complex projective structures (or more generally $(X, G)$-structures) has a natural complex symplectic structure $\omega_{G}$ which can be identified with the Goldman symplectic structure of the character variety via the holonomy map $h o l: \mathcal{C P}(S) \rightarrow \mathcal{X}(S, P S L(2, \mathbb{C}))$.

The deformation space of almost-Fuchsian structures $\mathcal{A F}(S)$ (introduced by Uhlenbeck in [Uhl83])) will be central in this article, it can be seen as an open subspace of either the character variety $\mathcal{X}\left(S, P S L_{2}(\mathbb{C})\right)$, Taubes' moduli space $\mathcal{H}$ or the space of complex projective structures $\mathcal{C P}(S)$. Using results of [Lou14] and an ad hoc notion of renormalized for almost-Fuchsian manifolds, we compare the Goldman symplectic structure $\omega_{G}$ of the character variety restricted to $\mathcal{A F}(S)$ with the symplectic structure $\omega_{\mathcal{H}}$ on $\mathcal{H}$ and, indirectly, the canonical symplectic structure $\omega_{\text {can }}$ on the cotangent bundle to Teichmüller space $T^{*} \mathcal{T}(S)$. We show in particular ${ }^{1}$

Theorem 4.9, In restriction to $\mathcal{A F}(S)$,

$$
\omega_{\mathcal{H}}=-\operatorname{Im}\left(\omega_{G}\right) .
$$

\footnotetext{
${ }^{1}$ Note that $\omega_{G}$ is a complex symplectic structure whereas $\omega_{\mathcal{H}}$ is a real symplectic structure.
} 
We explain the strategy in the remaining of this introduction.

First we compare the symplectic structure $\omega_{G}$ on $\mathcal{C P}(S)$ to the canonical symplectic structure $\omega_{\text {can }}$ on $T^{*} \mathcal{T}(S)$ under the Schwarzian parametrization of $\mathcal{C P}(S)$. This is thoroughly addressed in [Lou14]. Recall that there is a natural "forgetful" projection $p: \mathcal{C P}(S) \rightarrow \mathcal{T}(S)$ which assigns to a complex projective structure on $S$ its underlying complex structure. The Schwarzian parametrization turns $p: \mathcal{C P}(S) \rightarrow \mathcal{T}(S)$ into an affine holomorphic bundle modeled on $T^{*} \mathcal{T}(S)$. As a consequence there is an identification $\tau^{\sigma}: \mathcal{C P}(S) \stackrel{\sim}{\rightarrow} T^{*} \mathcal{T}(S)$, but it is not canonical: it depends on the choice of a "zero section" $\sigma: \mathcal{T}(S) \rightarrow \mathcal{C P}(S)$. The result proven in Lou14 that we will use is:

Theorem 2.5 ([Lou14, Corollary 6.13). Let $\sigma_{\mathcal{F}}: \mathcal{T}(S) \rightarrow \mathcal{C P}(S)$ be the Fuchsian section 2 , Then

$$
\left(\tau^{\sigma \mathcal{F}}\right)^{*} \omega_{\text {can }}=-i\left(\omega_{G}-p^{*} \omega_{W P}\right) .
$$

Next we turn our attention to Taubes' moduli space $\mathcal{H}$ and minimal surfaces in hyperbolic 3-manifolds. An observation going back to Hopf [Hop51] shows that if $\Sigma$ is a minimal surface in a hyperbolic 3-manifold $M$, then the second fundamental form $I I_{\Sigma}$ is the real part of a unique holomorphic quadratic differential $\varphi$ on $\Sigma$ with respect to the complex structure $\left[I_{\Sigma}\right]$ defined by the conformal class of the first fundamental form $I_{\Sigma}$. This defines a map $\Psi:(I, I I) \mapsto([I], \varphi)$ from $\mathcal{H}$ to $T^{*} \mathcal{T}(S)$ (recall that for $X \in \mathcal{T}(S), T_{X}^{*} \mathcal{T}(S)$ is naturally identified with the space of holomorphic quadratic differentials on the Riemann surface $X)$. The natural symplectic structure $\omega_{\mathcal{H}}$ on $\mathcal{H}$ can be described as $\omega_{T}=\operatorname{Re}\left(\Psi^{*} \omega_{\text {can }}\right)$.

Uhlenbeck ([Uhl83]) showed that if $M \approx S \times \mathbb{R}$ is equipped with a quasi-Fuchsian hyperbolic structure that happens to be almost-Fuchsian (see section 3.3), then there is a unique minimal embedding $S \hookrightarrow M$. Taking the first and second fundamental forms of the minimal surface defines an embedding of the almost-Fuchsian space $\mathcal{A F}(S)$ in the Taubes moduli space $\mathcal{H}$. The renormalized volume of $M$ is a "finite renormalization" of the otherwise infinite hyperbolic volume of $\left[\Sigma, \partial_{\infty}^{+} M\right)$, the end of $M$ comprised between the minimal surface $\Sigma$ and the ideal boundary $\partial_{\infty}^{+} M$, and it is related to equidistant foliations in this end. These ideas are made precise in sections 3 and 4 . Borrowing arguments mainly from [KS08] we prove the following theorem:

Theorem 4.8, Let $W: \mathcal{A F}(S) \rightarrow \mathbb{R}$ be the renormalized volume function on the almost-Fuchsian space and $\Psi: \mathcal{A F}(S) \rightarrow T^{*} \mathcal{T}(S)$ be the restriction to $\mathcal{A F}(S)$ of the map $\Psi: \mathcal{H} \rightarrow T^{*} \mathcal{T}(S)$ above. Then the differential of $W$, as a 1 -form on $\mathcal{A F}(S)$, is expressed as

$$
d W=-\frac{1}{4} \operatorname{Re}\left[\Psi^{*} \xi+\left(\tau^{\sigma \mathcal{F}}\right)^{*} \xi\right]
$$

where $\xi$ is the canonical one-form on the complex cotangent space $T^{*} \mathcal{T}(S)$ (so that $\left.\omega_{\text {can }}=d \xi\right)$.

\footnotetext{
${ }^{2} \sigma_{F}$ assigns to a complex structure on $S$ the uniformized Fuchsian projective structure, see section 2
} 
Theorem 4.9 then directly follows: just take the exterior derivative of this identity and use Theorem 2.5 above.

Note: The study and comparison of the affine cotangent symplectic structures on $\mathcal{C P}(S)$, Goldman's symplectic structure on $\mathcal{X}(S)$ and the "complex Hamiltonian picture" of $\mathcal{Q F}(S)$ is addressed in [Lou14]. Both articles are based on the author's $\mathrm{PhD}$ thesis ([Lou11]).

Structure of the paper: Section 2 discusses the affine cotangent symplectic structures on $\mathcal{C P}(S)$ and their relation with Goldman's symplectic structure on the character variety $\mathcal{X}(S)$. Section 3 reviews minimal surfaces in hyperbolic 3 -manifolds, Taubes' moduli space with its symplectic structure and almost-Fuchsian structures. Section 4 introduces renormalized volume for almost-Fuchsian manifolds and contains the proof of our main result.

Acknowledgments: This paper is based on part of the author's $\mathrm{PhD}$ thesis, which was supervised by Jean-Marc Schlenker. I wish to express my gratitude to Jean-Marc for his kind advice. I would also like to thank Steven Kerckhoff, Francis Bonahon, Bill Goldman, David Dumas, Jonah Gaster, Andy Sanders, Cyril Lecuire, Julien Marché, among others with whom I have had helpful discussions.

The research leading to these results has received funding from the European Research Council under the European Community's seventh Framework Programme (FP7/2007-2013)/ERC grant agreement.

\section{Complex projective structures and symplectic struc- tures}

Throughout this paper, $S$ will be a connected, oriented, smooth, closed surface with genus $g \geqslant 2$. We refer to [Lou14] for a more detailed exposition of this section.

\section{$2.1 \mathcal{C P}(S)$ and $\mathcal{T}(S)$}

A complex projective structure on $S$ is given as a maximal atlas of charts mapping open sets of $S$ into the complex projective line $\mathbb{C} \mathbf{P}^{1}$ such that the transition maps are (restrictions of) projective linear transformations (i.e. Möbius transformations of the Riemann sphere) ${ }^{3}$. The atlas is required to be compatible with the orientation and smooth structure on $S$. The group $\operatorname{Diff}^{+}(S)$ of orientation-preserving diffeomorphisms of $S$ naturally acts on the set of all complex projective structures on $S$,

\footnotetext{
${ }^{3}$ In terms of geometric structures (see e.g. [Thu97]), a complex projective structure is a $\left(\mathbb{C} \mathbf{P}^{1}, P S L_{2}(\mathbb{C})\right)$-structure.
} 
the quotient $\mathcal{C P}(S)$ by the subgroup $\operatorname{Diff}_{0}^{+}(S)^{4}$ is called the deformation space of complex projective structures on $S$.

Since a complex projective atlas is in particular a holomorphic atlas, a complex projective structure defines an underlying complex structure on $S$. This observation yields a "forgetful projection $p: \mathcal{C P}(S) \rightarrow \mathcal{T}(S)$, where the Teichmüller space $\mathcal{T}(S)=$ $\{$ complex structures on $S\} / \operatorname{Diff}_{0}^{+}(S)$ is the deformation space of complex structures on $S$.

Both $\mathcal{C P}(S)$ and $\mathcal{T}(S)$ are naturally complex manifolds, of dimensions $6 g-6$ and $3 g-3$ respectively. Let us recall in particular that the complex cotangent space $T_{X}^{*} \mathcal{T}(S)$ is naturally identified with the vector space $Q(X)$ of holomorphic quadratic differentials on the Riemann surface $X$ (for any $X \in \mathcal{T}(S)$ ). The "forgetful projection" $p: \mathcal{C P}(S) \rightarrow \mathcal{T}(S)$ is easily seen to be a holomorphic map. It is surjective (a section is given by uniformization) and its fibers are equipped with an affine structure, as explained in the subsequent paragraphs.

The Weil-Petersson metric on $\mathcal{T}(S)$ globalizes the Weil-Petersson product of holomorphic quadratic differentials, given by $\langle\varphi, \psi\rangle_{W P}=-\frac{1}{4} \int_{X} \varphi \cdot \sigma^{-1} \cdot \bar{\psi}$ for any $\varphi, \psi \in Q(X)$, where $\sigma^{-1}$ is the dual current of the area form $\sigma$ for the Poincaré metric $\left.\right|^{5}$ This defines a dual Hermitian metric $\langle\cdot, \cdot\rangle_{W P}$ on $\mathcal{T}(S)$, first shown to be Kähler by Ahlfors Ahl61 and Weil. The Kähler form of the Weil-Petersson metric on $\mathcal{T}(S)$ is the real symplectic form $\omega_{W P}=-\operatorname{Im}\langle\cdot, \cdot\rangle_{W P}$.

\subsection{Fuchsian and quasi-Fuchsian projective structures}

Whenever a Kleinian groun ${ }^{6} \Gamma$ acts freely and properly on some open subset $U$ of the complex projective line $\mathbb{C} \mathbf{P}^{1}$, the quotient surface $U / \Gamma$ inherits a complex projective structure. This gives a variety (but not all) of complex projective surfaces, called embedded projective structures.

Fuchsian projective structures are a fundamental example of embedded projective structures. Given a marked complex structure $X$, the uniformization theorem provides a representation $\rho: \pi_{1}(S) \rightarrow P S L_{2}(\mathbb{R})$ such that $X \approx \mathbb{H}^{2} / \rho\left(\pi_{1}(S)\right)$ as Riemann surfaces (where $\mathbb{H}^{2}$ is the upper half-plane). $\mathbb{H}^{2}$ can be seen as an open set (a disk) in $\mathbb{C} \mathbf{P}^{1}$ and the Fuchsian group $\rho\left(\pi_{1}(S)\right) \subset P S L_{2}(\mathbb{R})$ is in particular a Kleinian group, so the quotient $X \approx \mathbb{H}^{2} / \rho\left(\pi_{1}(S)\right)$ inherits a complex projective structure $Z$. This defines a section

$$
\sigma_{\mathcal{F}}: \mathcal{T}(S) \rightarrow \mathcal{C P}(S)
$$

to $p$, called the Fuchsian section. We call $\mathcal{F}(S):=\sigma_{\mathcal{F}}(\mathcal{T}(S))$ the (deformation) space of (standard) Fuchsian (projective) structures on $S$, it is an embedded copy of $\mathcal{T}(S)$ in $\mathcal{C P}(S)$.

\footnotetext{
${ }^{4} \operatorname{Diff}_{0}^{+}(S)$ is the identity component in $\operatorname{Diff}^{+}(S)$, consisting of diffeomorphisms that are homotopic to the identity.

${ }^{5}$ Recall that the Poincare metric is the unique conformal hyperbolic metric on $X$, given by uniformization. In a complex chart with values in the upper half-plane $z=x+i y: U \subset X \rightarrow \mathbb{H}^{2}$, the tensor product $-\frac{1}{4} \varphi \cdot \sigma^{-1} \cdot \bar{\psi}$ reduces to the classical expression $y^{2} \varphi(z) \overline{\psi(z)} d x \wedge d y$.

${ }^{6}$ i.e. a discrete subgroup of $P S L_{2}(\mathbb{C})$.
} 
Quasi-Fuchsian structures are another important class of embedded projective structures. Given two marked complex structures $\left.\left(X^{+}, X^{-}\right) \in \mathcal{T}(S) \times \mathcal{T}(\bar{S})\right]$. Bers' simultaneous uniformization theorem states that there exists a unique representation $\rho: \pi_{1}(S) \stackrel{\sim}{\rightarrow} \Gamma \subset P S L_{2}(\mathbb{C})$ up to conjugation such that:

- The limit set ${ }^{8} \Lambda$ is a Jordan curve. The domain of discontinuity $\Omega$ is then the disjoint union of two simply connected domains $\Omega^{+}$and $\Omega^{-}$. A such $\Gamma$ is called a quasi-Fuchsian group.

- As marked Riemann surfaces, $X^{+} \approx \Omega^{+} / \Gamma$ and $X^{-} \approx \Omega^{-} / \Gamma$.

Again, both Riemann surfaces $X^{+}$and $X^{-}$inherit embedded complex projective structures $Z^{+}$and $Z^{-}$by this construction. This defines a map $\beta=\left(\beta^{+}, \beta^{-}\right)$: $\mathcal{T}(S) \times \mathcal{T}(\bar{S}) \rightarrow \mathcal{C P}(S) \times \mathcal{C P}(\bar{S})$ which is a holomorphic section to $p \times p: \mathcal{C P}(S) \times$ $\mathcal{C P}(\bar{S}) \rightarrow \mathcal{T}(S) \times \mathcal{T}(\bar{S})$ by Bers' theorem.

In particular, when $X^{-} \in \mathcal{T}(\bar{S})$ is fixed, the map $\sigma_{X^{-}}:=\beta^{+}\left(\cdot, X^{-}\right): \mathcal{T}(S) \rightarrow$ $\mathcal{C P}(S)$ is a holomorphic section to $p$, called a Bers section. $\mathcal{Q F}(S):=\beta^{+}(\mathcal{T}(S) \times$ $\mathcal{T}(\bar{S})) \subset \mathcal{C P}(S)$ is called the (deformation) space of (standard) quasi-Fuchsian (projective) structures on $S$. It is an open neighborhood of $\mathcal{F}(S)$ in $\mathcal{C P}(S)$.

\subsection{The character variety}

References for this section include [Gol84], [HP04, Gol04] and [Dum09].

\section{The character variety and Goldman's symplectic structure}

Let $G=P S L_{2}(\mathbb{C})$ and $\mathcal{R}(S)$ be the set of group homomorphisms from $\pi:=\pi_{1}(S)$ to $G$. It has a natural structure of a complex affine algebraic set 9 , $G$ acts algebraically on $\mathcal{R}(S)$ by conjugation, and the character variety $\mathcal{X}(S)$ is defined as the quotient in the sense of invariant theory $\mathcal{X}(S)=\mathcal{R}(S) / G^{10}$. The points of $\mathcal{X}(S)$ are in one-to-one correspondence with the set of characters, i.e. complex-valued functions, defined on $\pi$, of the form $\gamma \in \pi \mapsto \operatorname{tr}^{2}(\rho(\gamma))$. The affine set $\mathcal{X}(S)$ splits into two irreducible components $\mathcal{X}(S)_{l} \cup \mathcal{X}(S)_{r}$, where elements of $\mathcal{X}(S)_{l}$ are characters of representations that lift to $S L(2, \mathbb{C})$.

\footnotetext{
${ }^{7}$ where $\bar{S}$ is the surface $S$ with reversed orientation

${ }^{8}$ The limit set $\Lambda=\Lambda(\Gamma)$ is defined as the complement in $\mathbb{C} \mathbf{P}^{1}$ of the domain of discontinuity $\Omega$, which is the maximal open set on which $\Gamma$ acts freely and properly. Alternatively, $\Lambda$ is described as the closure in $\mathbb{C} \mathbf{P}^{1}$ of the set of fixed points of elements of $\Gamma$.

${ }^{9}$ notably because there is an isomorphism $P S L_{2}(\mathbb{C}) \approx S O_{3}(\mathbb{C})$ (given by the adjoint representation of $P S L_{2}(\mathbb{C})$ on its Lie algebra $\left.\mathfrak{g}=\mathfrak{s l}_{2}(\mathbb{C})\right)$.

${ }^{10}$ Specifically, the action of $G$ on $\mathcal{R}(S)$ induces an action on the ring of regular functions $\mathbb{C}[\mathcal{R}(S)]$. Denote by $\mathbb{C}[\mathcal{R}(S)]^{G}$ the ring of invariant functions, it is finitely generated because $\mathcal{R}(S)$ is affine and $G$ is reductive. In fact, it is generated in this case $\left(G=P S L_{2}(\mathbb{C})\right)$ by a finite number of the complex valued functions on $\mathcal{R}(S)$ of the form $\rho \mapsto \operatorname{tr}^{2}(\rho(\gamma))$ (see [HP04]). $\mathcal{X}(S)$ is the affine set such that $\mathbb{C}[\mathcal{X}(S)]=\mathbb{C}[\mathcal{R}(S)]^{G}$.
} 
The set-theoretic quotient $\mathcal{R}(S) / G$ is rather complicated, but $G$ acts freely and properly on the subset $\mathcal{R}(S)^{s}$ of irreducible ${ }^{11}$ ("stable") representations, so that the quotient $\mathcal{R}(S)^{s} / G$ is a complex manifold. Furthermore, an irreducible representation is determined by its character, so that $\mathcal{X}(S)^{s}:=\mathcal{R}(S)^{s} / G$ embeds (as a Zariski-dense open subset) in the smooth locus of $\mathcal{X}(S)$. Its dimension is $6 g-6$.

By the general construction of Goldman, the character variety enjoys a complex symplectic structure. We refer to Gol84] for this construction, but here is how it is defined in a nutshell. At least for a stable point $[\rho] \in \mathcal{X}(S)^{s}$, the tangent space is identified in terms of group cohomology as the space $H^{1}\left(\pi, \mathfrak{g}_{\text {Ado }}\right)$. Here $\mathfrak{g}$ is the Lie algebra of $G$, seen as a $\pi$-module by Ad $\circ \rho: \pi \rightarrow \operatorname{Aut}(\mathfrak{g})$. Recall that the Lie algebra $\mathfrak{g}=\mathfrak{s l}_{2}(\mathbb{C})$ is equipped with its complex Killing form $B^{12}$. The symplectic pairing of tangent vectors is given by taking the cup-product in group cohomology with $B / 4$ as "coefficient pairing":

$$
H^{1}\left(\pi, \mathfrak{g}_{\text {Ado } \rho}\right) \times H^{1}\left(\pi, \mathfrak{g}_{\text {Ado } \rho}\right) \stackrel{\cup}{\rightarrow} H^{2}\left(\pi, \mathfrak{g}_{\text {Ado }} \otimes \mathfrak{g}_{\text {Ado } \rho}\right) \stackrel{B / 4}{\rightarrow} H^{2}(\pi, \mathbb{C}) \cong \mathbb{C} .
$$

By arguments of Goldman (Gol84]) following Atiyah-Bott ([AB83]), the complex 2 -form obtained this way is closed, in other words it is a complex symplectic form (at least on the smooth quasi-affine variety $\mathcal{X}(S)^{s}$ of stable points).

\section{Holonomy of projective structures}

Just like any geometric structure, a complex projective structure $Z$ defines a developing map and a holonomy representation (see e.g. [Thu97]). The developing map is a locally injective projective map $f: \tilde{Z} \rightarrow \mathbb{C} \mathbf{P}^{1}$ and it is equivariant with respect to the holonomy representation $\rho: \pi \rightarrow P S L_{2}(\mathbb{C})$ in the sense that $f \circ \gamma=\rho(\gamma) \circ f$ for any $\gamma \in \pi$.

Holonomy of complex projective structures defines a map

$$
\text { hol }: \mathcal{C P}(S) \rightarrow \mathcal{X}(S)
$$

which turns out to be a local biholomorphism, but neither injective nor a covering onto its image ([Hej75]). Nonetheless, we get a complex symplectic structure on $\mathcal{C P}(S)$ simply by pulling back that of $\mathcal{X}(S)^{s}$ by the holonomy map. Abusing notations, we will still call this symplectic structure $\omega_{G}{ }^{13}$. We will consider $\omega_{G}$ as the standard complex symplectic structure on $\mathcal{C P}(S)$.

The holonomy map is however injective in restriction to the space of standard quasi-Fuchsian projective structures $\mathcal{Q F}(S)$, so that $\mathcal{Q F}(S)$ and a fortiori $\mathcal{F}(S)$ are embedded in $\mathcal{X}(S)$. Note that the Fuchsian space $\mathcal{F}(S)$ is the deformation space of

\footnotetext{
${ }^{11}$ A representation $\rho: \pi \rightarrow P S L_{2}(\mathbb{C})$ is called irreducible if it fixes no point in $\mathbb{C} \mathbf{P}^{1}$.

${ }^{12}$ It is a non-degenerate complex bilinear symmetric form preserved by $G$ under the adjoint action, explicitly given by $B(u, v)=4 \operatorname{tr}(u v)$ where $u, v \in \mathfrak{s l}_{2}(\mathbb{C})$ are represented by trace-free $2 \times 2$ matrices.

${ }^{13}$ Alternatively, one could directly define $\omega_{G}$ on $\mathcal{C P}(S)$ in terms of the exterior product of 1 forms with values in some flat bundle. The tangent space to $\mathcal{C P}(S)$ at a point $Z$ can be identified as $T_{Z} \mathcal{C P}(S)=\check{H}^{1}\left(Z, \Xi_{Z}\right)$, where $\Xi_{Z}$ is the sheaf of projective vector fields on $Z$.
} 
hyperbolic structures on $S$, under the holonomy map it is identified as the component of discrete and faithful representations in the real character variety $\mathcal{X}\left(S, P S L_{2}(\mathbb{R})\right)$. In Gol84, Goldman also shows that the symplectic form $\omega_{G}$ restricts to the WeilPetersson Kähler form $\omega_{W P}$ on this component. More precisely, this is stated as follows in our setting:

Theorem 2.1 (Goldman Gol84]). Recall that $\sigma_{\mathcal{F}}: \mathcal{T}(S) \rightarrow \mathcal{C P}(S)$ denotes the Fuchsian section. Then

$$
\left(\sigma_{\mathcal{F}}\right)^{*} \omega_{G}=\omega_{W P} .
$$

\subsection{Schwarzian parametrization of $\mathcal{C P}(S)$ and the affine cotangent symplectic structures}

\section{Schwarzian parametrization}

The Schwarzian parametrization turns the space $\mathcal{C P}(S)$ with its projection $p$ : $\mathcal{C P}(S) \rightarrow \mathcal{T}(S)$ into a holomorphic affine bundle modeled on the holomorphic cotangent vector bundle $\pi: T^{*} \mathcal{T}(S) \rightarrow \mathcal{T}(S)$. We very briefly sketch how this works, and refer to e.g. Dum09 for a detailed exposition (the details of this construction will not be required for the purpose of this paper).

Given a locally injective holomorphic function $f: Z_{1} \rightarrow Z_{2}$ where $Z_{1}$ and $Z_{2}$ are complex projective surfaces, define the osculating map $\tilde{f}$ to $f$ at a point $m \in Z_{1}$ as the germ of a (locally defined) projective map that has the best possible contact with $f$ at $m$. In some sense, one can take a flat covariant derivative $\nabla \tilde{f}$ and identify it as holomorphic quadratic differential $\mathcal{S}(f) \in Q(X)$, called the Schwarzian derivative of $f$.

Let $X$ be a fixed point in $\mathcal{T}(S)$ and $P(X):=p^{-1}(\{X\})$ the set of marked projective structures on $S$ whose underlying complex structure is $X$. Given $Z_{1}$, $Z_{2} \in P(X)$, the identity $\operatorname{map} \operatorname{id}_{S}: Z_{1} \rightarrow Z_{2}$ is holomorphic but not projective if $Z_{1} \neq Z_{2}$. Taking its Schwarzian derivative $\varphi=\mathcal{S}\left(\mathrm{id}_{S}\right) \in Q(X)$ accurately measures the "difference $Z_{2}-Z_{1}$ " between the two projective structures $Z_{1}$ and $Z_{2}$. It turns out that the properties of the Schwarzian derivative ensure that $Z_{1}, Z_{2} \in P(X) \mapsto$ $\varphi \in Q(X)$ indeed equips $P(X)$ with the structure of a complex affine space modeled on the vector space $Q(X)$.

Recall that $Q(X)$ is also identified with the complex cotangent space $T_{X}^{*} \mathcal{T}(S)$. As a result of this discussion, $p: \mathcal{C P}(S) \rightarrow \mathcal{T}(S)$ is an affine holomorphic bundle modeled on the holomorphic cotangent vector bundle $T^{*} \mathcal{T}(S)$. As a consequence, $\mathcal{C P}(S)$ can be identified with $T^{*} \mathcal{T}(S)$ by choosing a "zero section" $\sigma: \mathcal{T}(S) \rightarrow \mathcal{C P}(S)$. Explicitly, we get an isomorphism of complex affine bundles $\tau^{\sigma}: Z \mapsto Z-\sigma(p(Z))$ such that $\tau^{\sigma} \circ \sigma$ is the zero section to $\pi: T^{*} \mathcal{T}(S) \rightarrow \mathcal{T}(S)$. It is an isomorphism of holomorphic bundles whenever $\sigma$ is a holomorphic section to $p$, such as a Bers section. 


\section{Cotangent affine symplectic structures}

Recall that for any complex manifold $M$ (in particular $M=\mathcal{T}(S)$ ), the total space of its holomorphic cotangent bundle $T^{*} M$ is equipped with a canonical holomorphic 1 -form $\xi$ and a canonical complex symplectic structure $\omega_{\text {can }}=d \xi$.

As we have seen in the previous paragraph, any choice of a "zero section" $\sigma$ : $\mathcal{T}(S) \rightarrow \mathcal{C P}(S)$ yields an affine isomorphism $\tau^{\sigma}: \mathcal{C P}(S) \stackrel{\sim}{\rightarrow} T^{*} \mathcal{T}(S)$. We can use this to pull back the canonical symplectic structure of $T^{*} \mathcal{T}(S)$ on $\mathcal{C} \mathcal{P}(S)$ : define

$$
\omega^{\sigma}:=\left(\tau^{\sigma}\right)^{*} \omega_{\text {can }} .
$$

How is $\omega^{\sigma}$ affected by the choice of the "zero section" $\sigma$ ? A small computation (see [Lou14]) shows that:

Proposition 2.2. For any two sections $\sigma_{1}$ and $\sigma_{2}$ to $p: \mathcal{C P}(S) \rightarrow \mathcal{T}(S)$,

$$
\omega^{\sigma_{2}}-\omega^{\sigma_{1}}=-p^{*} d\left(\sigma_{2}-\sigma_{1}\right)
$$

where $\sigma_{2}-\sigma_{1}$ is the "affine difference" between $\sigma_{2}$ and $\sigma_{1}$, it is a 1 -form on $\mathcal{T}(S)$.

Now, McMullen proved in McM00] the following theorem:

Theorem 2.3 (McMullen [McM00]). If $\sigma$ is any Bers section, then

$$
d\left(\sigma_{\mathcal{F}}-\sigma\right)=-i \omega_{W P} .
$$

A consequence of this is that if $\sigma$ is chosen among Bers sections, $\omega^{\sigma}$ does not depend on $\sigma$. Moreover, $\omega^{\sigma}$ is a complex symplectic form on $\mathcal{C P}(S)$ which restricts to $-i \omega_{W P}$ on the Fuchsian slice $\mathcal{F}(S)$, where $\omega_{W P}$ is the Weil-Petersson Kähler form on $\mathcal{T}(S)$. On the other hand, the standard symplectic structure $\omega_{G}$ is a complex symplectic structure on $\mathcal{C P}(S)$ which restricts to $\omega_{W P}$ on the Fuchsian slice (see 6). An analytic continuation argument ensures that:

Theorem 2.4 ([Lou14], Theorem 6.10). If $\sigma: \mathcal{T}(S) \rightarrow \mathcal{C P}(S)$ is any Bers section, then

$$
\omega^{\sigma}=-i \omega_{G} .
$$

We also get the expression of the 2 -form $\omega^{\sigma_{\mathcal{F}}}$ :

Corollary 2.5 ([Lou14], Corollary 6.13). Let $\sigma_{\mathcal{F}}: \mathcal{T}(S) \rightarrow \mathcal{C P}(S)$ be the Fuchsian section. Then

$$
\omega^{\sigma_{\mathcal{F}}}=-i\left(\omega_{G}-p^{*} \omega_{W P}\right) .
$$

We refer to [Lou14 for details. 


\section{Minimal surfaces in hyperbolic 3-manifolds, Taubes' moduli space and almost-Fuchsian structures}

Minimal surfaces in hyperbolic 3-manifolds have generated a lot of attention, with important contributions by Uhlenbeck ([Uhl83]) and more recently Taubes ([Tau04]). In this paper, a primary source of inspiration was the work of Krasnov-Schlenker ([KS07], [KS08], [KS07]).

\subsection{Minimal surfaces in hyperbolic 3-manifolds}

Extrinsic invariants of surfaces in hyperbolic 3-manifolds and the GaussCodazzi equations

Consider an oriented immersed surface $\Sigma$ in an oriented hyperbolic 3-manifold $M$. Denote by $g$ the hyperbolic metric on $M$ and $\bar{\nabla}$ its Levi-Civita connection. The following are classical extrinsic invariants of $\Sigma$ :

- The first fundamental form $I$ is the Riemannian metric on $\Sigma$ induced by the hyperbolic metric $g$. Let $\nabla$ denote its Levi-Civita connection and $K$ its curvature.

- The shape operator $B \in \operatorname{End}(T \Sigma)$ is defined by $B v:=-\bar{\nabla}_{v} n$, where $n$ is the positively oriented unit normal vector field to $\Sigma$. It is self-adjoint with respect to $I$. The mean curvature is defined by $H:=\operatorname{tr}(B)$.

- The second fundamental form $I I$ is the symmetric bilinear form associated to $B$ with respect to $I: I I(u, v):=I(B u, v)=I(u, B v)$. We use the following notation convention: $B=I^{-1} I I$.

- The third fundamental form III is the symmetric bilinear form defined by $I I I(u, v)=I(B u, B v)$.

These satisfy the Gauss-Codazzi equations on $\Sigma$ :

$$
\begin{cases}\operatorname{det} B=K+1 & \text { (Gauss equation) } \\ d^{\nabla} B=0 & \text { (Codazzi equation) }\end{cases}
$$

where $B$ is seen as a $T \Sigma$-valued one-form in the Codazzi equation and $d^{\nabla}$ is the extension of the exterior derivative using the connection $\nabla$.

Conversely, the "fundamental theorem of surface theory" states that if $I$ is a Riemannian metric on a surface $S$ and $I I$ is a symmetric bilinear form such that $I$ and $I I$ satisfy the Gauss-Codazzi equations, then there exists an isometric immersion of $(S, I)$ in a possibly non-complete hyperbolic 3 -manifold $M$ such that $I I$ is the second fundamental form of the immersed surface. 


\section{Minimal surfaces in hyperbolic 3-manifolds and holomorphic quadratic differentials}

A minimal surface in a hyperbolic 3 -manifold $M$ is a minimally ${ }^{14}$ isometrically immersed Riemannian surface $(S, I)$ in $M$. By the "fundamental theorem of surface theory", this is equivalent to the three following conditions on the extrinsic invariants of the immersed surface:

$$
\left\{\begin{array}{l}
\operatorname{det} B=K+1 \\
d^{\nabla} B=0 \\
H=0 .
\end{array}\right.
$$

The following lemma was first discovered by Hopf and is quite straightforward to prove but it provides a surprising relation between minimal surfaces and holomorphic quadratic differentials:

Lemma 3.1 ([Hop51]). Let $S$ be an oriented surface equipped with a Riemannian metric $I$ and a symmetric bilinear form II on $S$. Let $B:=I^{-1} I I$. Consider the conformal class $[I]$, so that $X:=(S,[I])$ is a Riemann surface.

(i) II is the real part of a (unique) smooth quadratic differential $\varphi$ if and only if $\operatorname{tr}(B)=0$.

(ii) If (i) holds, then $\varphi$ is holomorphic on $X$ if and only if $d^{\nabla} B=0$.

In particular, any embedded minimal surface $S \hookrightarrow M$ in a hyperbolic 3-manifold defines a Riemann surface $X:=(S,[I])$ and a holomorphic quadratic differential $\varphi \in Q(X)$, i.e. a point in the holomorphic cotangent of the Teichmüller space of $S$ (see section 2.1).

\subsection{Taubes' moduli space}

A minimal hyperbolic germ is a pair $(I, I I)$ where $I$ is a (smooth) Riemannian metric on $S$ and $I I$ is a symmetric bilinear form on on $S$ such that $\operatorname{det} B=K+1, d^{\nabla} B=0$ and $H=0$, where the notations correspond to the ones above: $K$ is the curvature of $I, B=I^{-1} I I$ (meaning that $B$ is the $I$-self-adjoint endomorphism of $T S$ associated to $I I), \nabla$ is the Levi-Civita connection of $I, d^{\nabla}$ is the extension of $\nabla$ to $T S$-valued 1forms and $H=\operatorname{tr}(B)$. Alternatively, one could define a minimal hyperbolic germ as a pair $(I, \varphi)$, where $I$ is a Riemannian metric on $S$ and $\varphi$ is a holomorphic quadratic differential (with respect to the complex structure on $S$ determined by the conformal class of $I$ and the orientation of $S$ ) such that $-\|\varphi\|_{I}^{2}=K+1$. The relation with the previous definition is just $I I=\operatorname{Re}(\varphi)$, and one can check that $-\|\varphi\|_{I}^{2}=\operatorname{det} B$. More geometrically, a minimal hyperbolic germ is a pair $(I, I I)$ such that $I I$ is the

\footnotetext{
${ }^{14}$ In the sense that it is an extremal of the area functional. Equivalently, the mean curvature $H$ of the immersed surface vanishes identically. We refer to e.g. Che00 for general background on minimal submanifolds of Riemannian manifolds.
} 
second fundamental form of a minimal isometric embedding of $(S, I)$ into a (possibly non-complete) hyperbolic 3-manifold.

Taubes' moduli space is then defined as the quotient of the set of all hyperbolic germs by the natural action of the group $\operatorname{Diff}_{0}^{+}(S)$. In [Tau04, Taubes shows that this quotient only has smooth points (in a reasonable sense), so that $\mathcal{H}$ is a smooth manifold (of dimension $12 g-12$ ).

The map which assigns to a minimum hyperbolic germ $(I, I I)$ the couple $(X, \varphi)$ where $X$ is the complex structure on $S$ determined by the conformal class of $I$ and the orientation of $S$ and $\varphi \in Q(X)$ is the holomorphic quadratic differential on $X$ such that $I I=\operatorname{Re}(\varphi)$ descends to the quotient as a well-defined smooth map $\Psi: \mathcal{H} \rightarrow T^{*} \mathcal{T}(S)$ (recall that $\left.T_{X}^{*} \mathcal{T}(S) \approx Q(X)\right)$.

Taubes also shows that $\mathcal{H}$ naturally enjoys a real symplectic structure $\omega_{\mathcal{H}}$, obtained by a symplectic reduction of the space $T^{*} \operatorname{Met}(S)$ equipped with its canonical symplectic structure (here $\operatorname{Met}(S)$ denotes the space of smooth Riemannian metrics on $S$ ). Conveniently, this symplectic structure can alternatively be described as $\omega_{\mathcal{H}}=\operatorname{Re}\left(\Psi^{*} \omega_{\text {can }}\right)^{15}$.

\subsection{Almost-Fuchsian structures}

A minimal hyperbolic germ $(I, I I)$ is called almost-Fuchsian when $\|I I\|_{I}^{2}<2$ everywhere ${ }^{16}$. Said differently, $I I$ is the second fundamental form for a minimal isometric embedding of $\Sigma$ in a hyperbolic 3-manifold such that its principal curvatures are everywhere in $(-1,1)$. The almost-Fuchsian space $\mathcal{A} \mathcal{F}(S)$ is defined as the subspace of $\mathcal{H}$ of classes of almost-Fuchsian minimal hyperbolic germs.

This definition is motivated by the observation of Uhlenbeck [Uhl83] that the germ of the hyperbolic metric associated to an almost-Fuchsian germ can be extended to a complete hyperbolic metric on $S \times \mathbb{R}$. More precisely, if $(I, I I)$ is an almostFuchsian minimal hyperbolic germ, then there is a unique quasi-Fuchsian 3-manifold $M{ }^{17}$ up to isometry with a minimal isometric embedding $(S, I) \hookrightarrow M$ giving $I I$ as the second fundamental form.

As a consequence, almost-Fuchsian space $\mathcal{A F}(S) \subset \mathcal{H}$ embeds as an open set of $\mathcal{Q F}(S)$. Recall that the space of quasi-Fuchsian structures $\mathcal{Q F}(S)$ can be seen either as a subspace of the space of complex projective structures $\mathcal{C P}(S)$ or the character variety $\mathcal{X}(S)$. In turn, we will (somewhat abusively) think of almostFuchsian structures as living either in $\mathcal{H}, \mathcal{C P}(S)$ or $\mathcal{X}(S)$, depending on the context.

Note that the Fuchsian space $\mathcal{F}(S)$ corresponds to the "Fuchsian minimal hyperbolic germs", i.e. of the form $(I, 0)$. The map $\Psi: \mathcal{H} \mapsto T^{*} \mathcal{T}(S)$ restricts to

\footnotetext{
15 Taubes' definition of $\omega_{\mathcal{H}}$ probably differs from ours by a factor 2 , because the canonical real symplectic structure on the real cotangent bundle $T_{\mathbb{R}}{ }^{*} \mathcal{T}(S)$ and the real part of the canonical complex symplectic structure on the holomorphic cotangent bundle $T^{*} \mathcal{T}(S)$ differ by a factor 2 under the usual identification $T_{\mathbb{R}}{ }^{*} \mathcal{T}(S) \approx T^{*} \mathcal{T}(S)$.

${ }^{16}$ or equivalently $\operatorname{det} B>-1$, or $\|\varphi\|_{I}^{2}<1$. See previous paragraph for the notations $B$ and $\varphi$.

${ }^{17}$ A quasi-Fuchsian 3-manifold is a manifold isometric to $\mathbb{H}^{3} / \Gamma$ where $\Gamma$ is a quasi-Fuchsian group as in 2.2 .
} 
"the identity" on the space of Fuchsian germs. In other words, it identifies the space of Fuchsian germs $(\approx \mathcal{F}(S))$ with the zero section of $T^{*} \mathcal{T}(S)(\approx \mathcal{T}(S) \approx \mathcal{F}(S)$ by uniformization).

\section{Renormalized volume of almost-Fuchsian manifolds}

In this section we introduce an ad hoc notion of renormalized volume for almostFuchsian manifolds. Studying its infinitesimal variations will enable us to compare the symplectic structure $\omega_{\mathcal{H}}$ on the almost-Fuchsian space to the symplectic structures encountered in section 2. We refer to [KS08] and [KS12] for a systematic presentation of the renormalized volume of hyperbolic 3-manifolds.

\subsection{Equidistant foliations in quasi-Fuchsian 3-manifolds}

Let $M$ be a quasi-Fuchsian 3-manifold. Consider a smooth embedded surface $S_{0}$ such that the normal exponential map induces an isotopic deformation of $S_{0}$ on the ideal boundary component $\partial_{\infty}^{+} M$. In particular, $S_{0}$ disconnects $M$ (or equivalently $\left.\hat{M}=M \cup \partial_{\infty} M\right)$ into two connected components, each one containing a boundary component. Denote by $\left[S_{0}, \partial_{\infty}^{+} M\right]$ (resp. $\left.\left[\partial_{\infty}^{-} M, S_{0}\right]\right)$ the connected component of $\hat{M}$ containing $\partial_{\infty}^{+} M$ (resp. $\left.\partial_{\infty}^{-} M\right)$. Let $S_{\rho}$ be the set of points at distance $\rho \geqslant 0$ of $S_{0}$ in $\left[S_{0}, \partial_{\infty}^{+} M\right]$. We assume now that $S_{0}$ is convex, in the sense that $\left.] \partial_{\infty}^{-} M, S_{0}\right]$ is geodesically convex in $M$. The nearest-point projection $\kappa:\left[S_{0}, \partial_{\infty}^{+} M\left[\rightarrow S_{0}\right.\right.$ is welldefined and smooth, and it admits a natural extension to $\partial_{\infty}^{+} M$. It is easy to see that $S_{\rho}$ is a smooth embedded surface, obtained as the image of a section $u_{\rho}: S_{0} \rightarrow S_{\rho}$ to $h^{18}$ (including in the case $\rho=+\infty$, with $S_{\infty}=\partial_{\infty}^{+} M$ ). The 1-parameter family $\left(S_{\rho}\right)_{\rho \geqslant 0}$ is called an equidistant foliation of the topological end $\partial^{+} \hat{M}$, it is a smooth foliation of $\left[S_{0}, \partial_{\infty}^{+} M\right]$ by equidistant surfaces. Two equidistant foliations $\left(S_{\rho}\right)_{\rho \geqslant 0}$ and $\left(S_{\rho}^{\prime}\right)_{\rho \geqslant 0}$ are considered equivalent if $S_{0}=S_{\rho}^{\prime}$ for some $\rho>0$ or vice-versa.

Let $\left(S_{\rho}\right)_{\rho \geqslant 0}$ be an equidistant foliation as above. For any $\rho \geqslant 0$, the map $f_{\rho}=$ $u_{\rho} \circ u_{\infty}{ }^{-1}$ is a smooth identification $f_{\rho}: S_{\infty} \stackrel{\sim}{\rightarrow} S_{\rho}$. Let $I_{\rho}:=f_{\rho}{ }^{*} I_{S_{\rho}}$ where $I_{S_{\rho}}$ is the first fundamental form on $S_{\rho}$, and define $I I_{\rho}$ and $I I I_{\rho}$ similarly. Writing down the differential equation satisfied by $I_{\rho}$ and integrating it, one shows (see [KS07]):

Proposition 4.1. For any $\rho, h>0$,

$$
I_{\rho+h}=\left(\cosh ^{2} h\right) I_{\rho}+2(\sinh h \cosh h) I I_{\rho}+\left(\sinh ^{2} h\right) I I I_{\rho} .
$$

The following easily follows:

\section{Proposition 4.2.}

- $I_{\rho} \sim_{\rho \rightarrow \infty} \frac{e^{2 \rho}}{2} I^{*}$, where $I^{*}:=\frac{1}{2}\left(I_{0}+2 I I_{0}+I I I_{0}\right)$.

\footnotetext{
${ }^{18}$ Note that $\kappa$ is a right inverse to the normal exponential map, and $u_{\rho}$ is just the time- $\rho$ normal exponential map.
} 
- For any $\rho \geqslant 0, I^{*}=\frac{e^{-2 \rho}}{2}\left(I_{\rho}+2 I I_{\rho}+I I I_{\rho}\right)$.

- $I I^{*}:=\frac{1}{2}\left(I_{\rho}-I I I_{\rho}\right)$ does not depend on $\rho$.

A standard argument shows that $I^{*}$ must belong to the conformal class of the ideal boundary $\partial_{\infty}^{+} M$. Furthermore, a theorem first proved by C. Epstein (see also [HR93]) says that:

Theorem 4.3 (C. Epstein). Given any metric $g$ in the conformal class of $\partial_{\infty}^{+} M$, there is a unique equidistant foliation up to equivalence $\left(S_{\rho}\right)_{\rho \geqslant 0}$ such that $I^{*}=g$.

\subsection{Definition of the renormalized volume}

Suppose now that $M$ is almost-Fuchsian, so that it contains a unique minimal surface $\Sigma$. Let $\left(S_{\rho}\right)_{\rho \geqslant 0}$ be the unique equidistant foliation associated to the Poincare metric on $\partial_{\infty}^{+} M \stackrel{19}{19}$. Denote by $N_{\rho}=\left[\Sigma, S_{\rho}\right]$ be the compact connected component of $M \backslash$ $\left(\Sigma \cup S_{\rho}\right)$ and by $V\left(N_{\rho}\right)$ its hyperbolic volume. A direct calculation shows that:

Proposition 4.4. The number

$$
W=V\left(N_{\rho}\right)-\frac{1}{4}\left(\int_{S_{\infty}} H_{\rho} d a_{\rho}\right)-2 \pi(g-1) \rho
$$

does not depend on $\rho$. NB: here $H_{\rho}$ is the mean curvature $H_{\rho}=\operatorname{tr}\left(I_{\rho}^{-1} I I_{\rho}\right)$ and $d a_{\rho}$ is the area element for the metric $I_{\rho}$.

$W$ will be called the renormalized volume of the almost-Fuchsian manifold $M$. Renormalized volume thus defines a function $W: \mathcal{A F}(S) \rightarrow \mathbb{R}$.

\subsection{Variation of the renormalized volume, comparing symplectic structures}

Next we want to compute the variation of the renormalized volume under an infinitesimal variation of the hyperbolic metric (in other words we want to compute the differential $d W$ ).

The following formula for the variation of volume was proved by Rivin-Schlenker [RS99]:

Theorem 4.5 (Rivin-Schlenker [RS99]). Let $M$ be a convex cocompact hyperbolic 3-manifold and let $N \subset M$ be a convex compact subset of $M$ with smooth boundary. Under an infinitesimal deformation of the hyperbolic metric on $M$,

$$
2 \delta V(N)=\int_{\partial N}\left(\delta H+\frac{1}{2}\langle\delta I, I I\rangle_{I}\right) d a_{I} .
$$

We use this to show:

\footnotetext{
${ }^{19}$ i.e. $I_{*}=\frac{e^{-2 \rho}}{2}\left(I_{\rho}+2 I I_{\rho}+I I I_{\rho}\right)($ for any $\rho)$ is the unique conformal hyperbolic metric on $\partial_{\infty}^{+} M$.
} 
Theorem 4.6. Let $M$ be an almost-Fuchsian manifold. Under an infinitesimal deformation of the hyperbolic metric on $M$, the variation of the renormalized volume is given by

$$
\delta W=-\frac{1}{4} \int_{S_{\infty}}\left\langle I I_{\Sigma}, \delta I_{\Sigma}\right\rangle_{I_{\Sigma}} d a_{\Sigma}-\frac{1}{4} \int_{S_{\infty}}\left\langle I I_{0}^{*}, \delta I^{*}\right\rangle_{I^{*}} d a^{*}
$$

where $I I_{0}^{*}$ is the trace-free part of $I I^{*}$ (with respect to $I^{*}$ ).

Proof. We only give an outline of the proof; most of the calculations needed have already been done in [KS08]. Following the definition of $W$, one has

$$
\delta W=\delta V\left(N_{\rho}\right)-\frac{1}{4}\left(\int_{S_{\infty}} \delta H_{\rho} d a_{\rho}\right)-\frac{1}{4}\left(\int_{S_{\infty}} H_{\rho} \delta\left(d a_{\rho}\right)\right)
$$

Using the formula 16, the variation of $V\left(N_{\rho}\right)$ is given by

$$
\delta V\left(N_{\rho}\right)=-\frac{1}{4}\left(\int_{S_{\infty}}\left\langle\delta I_{\Sigma}, I I_{\Sigma}\right\rangle_{I_{\Sigma}}\right) d a_{\Sigma}+\frac{1}{2}\left(\int_{S_{\infty}} \delta H_{\rho}+\frac{1}{2}\left\langle\delta I_{\rho}, I I_{\rho}\right\rangle_{I_{\rho}} d a_{\rho}\right) .
$$

The variation of the mean curvature $H$ on a surface is $\delta H=-\langle\delta I, I I\rangle_{I}+\langle I, \delta I I\rangle_{I}$, and the variation of the area element is given by $\delta(d a)=\frac{1}{2}\langle\delta I, I\rangle_{I} d a$. Putting all this together, one finds

$$
\delta W=-\frac{1}{4}\left(\int_{S_{\infty}}\left\langle\delta I_{\Sigma}, I I_{\Sigma}\right\rangle_{I_{\Sigma}}\right) d a_{\Sigma}+\frac{1}{4}\left(\int_{S_{\infty}}\left\langle\delta I I_{\rho}-\frac{H_{\rho}}{2} \delta I_{\rho}, I_{\rho}\right\rangle_{I_{\rho}} d a_{\rho}\right) .
$$

A tedious but straightforward calculation done in [KS08] (Corollary 6.2) shows that for any foliation $\left(S_{\rho}\right)_{\rho \geqslant 0}$,

$$
\int_{S_{\infty}}\left\langle\delta I I_{\rho}-\frac{H_{\rho}}{2} \delta I_{\rho}, I_{\rho}\right\rangle_{I_{\rho}} d a_{\rho}=-\int_{S_{\infty}} \delta H^{*}+\left\langle\delta I^{*}, I I_{0}^{*}\right\rangle_{I^{*}} d a^{*}
$$

It is also shown there (Remark 5.4) that $H^{*}=-K^{*}$. However, we have chosen $I^{*}$ such that $K^{*}=-1$, it follows that $\delta H^{*}=0$.

We need this last ingredient, proven in [KS08]:

Proposition 4.7 (Krasnov-Schlenker [KS08]). Let $Z \in \mathcal{Q F}(S)$ be a quasi-Fuchsian structure and $M$ be the associated quasi-Fuchsian 3-manifold. Let $\left(S_{\rho}\right)_{\rho \geqslant 0}$ be the unique equidistant foliation associated to the Poincaré metric on $\partial_{\infty}^{+} M$ and let $I I_{0}^{*}$ $\left(=I I^{*}-I^{*}\right)$ be the trace-free part of $I I^{*}$ as above. Then

$$
I I_{0}^{*}=\operatorname{Re}\left(\tau^{\sigma_{\mathcal{F}}}(Z)\right)
$$


Recall that $\tau^{\sigma_{\mathcal{F}}}: \mathcal{C P}(S) \rightarrow T^{*} \mathcal{T}(S)$ is the identification of holomorphic affine bundles (given by the Schwarzian parametrization) when one chooses the Fuchsian section $\sigma_{\mathcal{F}}: \mathcal{T}(S) \rightarrow \mathcal{C P}(S)$ as the "zero section" of $\mathcal{C P}(S)$ (see paragraph 2.4). In other words, $\tau^{\sigma_{\mathcal{F}}}(Z)$ is the holomorphic quadratic differential on $\partial_{\infty}^{+} M$ given by the Schwarzian of the projective structure $Z$ on $\partial_{\infty}^{+} M$ relative to the uniformized projective structure.

As a consequence of this proposition and the previous theorem, we obtain:

Theorem 4.8. Let $W: \mathcal{A F}(S) \rightarrow \mathbb{R}$ be the renormalized volume function on the almost-Fuchsian space, $\Psi: \mathcal{A F}(S) \rightarrow T^{*} \mathcal{T}(S)$ be the map defined in paragraph 3.2. Then

$$
d W=-\frac{1}{4} \operatorname{Re}\left[\Psi^{*} \xi+\left(\tau^{\sigma_{\mathcal{F}}}\right)^{*} \xi\right]
$$

where $\xi$ is the canonical one-form on the complex cotangent space $T^{*} \mathcal{T}(S)$.

Note that this is an equality of real 1-forms on $\mathcal{A F}(S)$. Taking the exterior derivative again yields $0=-\frac{1}{4} \operatorname{Re}\left[\Psi^{*} \omega_{\text {can }}+\left(\tau^{\sigma_{\mathcal{F}}}\right)^{*} \omega_{\text {can }}\right]$. Recall that $\omega_{\mathcal{H}}=\operatorname{Re}\left(\Psi^{*} \omega_{\text {can }}\right)$ (see paragraph 3.2) and $\left(\tau^{\sigma \mathcal{F}}\right)^{*} \omega_{\text {can }}=-i\left(\omega_{G}-p^{*} \omega_{W P}\right)$ (Corollary 2.5). The identification of symplectic structures follows:

Theorem 4.9. On the almost-Fuchsian space $\mathcal{A F}(S)$,

$$
\omega_{\mathcal{H}}=-\operatorname{Im}\left(\omega_{G}\right) .
$$

\section{References}

[AB83] M. F. Atiyah and R. Bott. The Yang-Mills equations over Riemann surfaces. Philos. Trans. Roy. Soc. London Ser. A, 308(1505):523-615, 1983.

[Ahl61] Lars V. Ahlfors. Some remarks on Teichmüller's space of Riemann surfaces. Ann. of Math. (2), 74:171-191, 1961.

[Che00] Bang-Yen Chen. Riemannian submanifolds. In Handbook of differential geometry, Vol. I, pages 187-418. North-Holland, Amsterdam, 2000.

[Dum09] David Dumas. Complex projective structures. In Handbook of Teichmüller theory. Vol. II, volume 13 of IRMA Lect. Math. Theor. Phys., pages 455508. Eur. Math. Soc., Zürich, 2009.

[Gol84] William M. Goldman. The symplectic nature of fundamental groups of surfaces. Adv. in Math., 54(2):200-225, 1984.

[Gol04] William M. Goldman. The complex-symplectic geometry of SL(2, C)characters over surfaces. In Algebraic groups and arithmetic, pages 375407. Tata Inst. Fund. Res., Mumbai, 2004. 
[Hej75] Dennis A. Hejhal. Monodromy groups and linearly polymorphic functions. Acta Math., 135(1):1-55, 1975.

[Hop51] Heinz Hopf. Über Flächen mit einer Relation zwischen den Hauptkrümmungen. Math. Nachr., 4:232-249, 1951.

[HP04] Michael Heusener and Joan Porti. The variety of characters in $\mathrm{PSL}_{2}(\mathbb{C})$. Bol. Soc. Mat. Mexicana (3), 10(Special Issue):221-237, 2004.

[HR93] Craig D. Hodgson and Igor Rivin. A characterization of compact convex polyhedra in hyperbolic 3-space. Invent. Math., 111(1):77-111, 1993.

[KS07] Kirill Krasnov and Jean-Marc Schlenker. Minimal surfaces and particles in 3-manifolds. Geom. Dedicata, 126:187-254, 2007.

[KS08] Kirill Krasnov and Jean-Marc Schlenker. On the renormalized volume of hyperbolic 3-manifolds. Comm. Math. Phys., 279(3):637-668, 2008.

[KS12] Kirill Krasnov and Jean-Marc Schlenker. The Weil-Petersson metric and the renormalized volume of hyperbolic 3-manifolds. In Handbook of Teichmüller theory. Volume III, volume 17 of IRMA Lect. Math. Theor. Phys., pages 779-819. Eur. Math. Soc., Zürich, 2012.

[Lou11] Brice Loustau. The symplectic geometry of the deformation space of complex projective structures over a surface. $\mathrm{PhD}$ thesis, University of Toulouse III, Toulouse, 2011.

[Lou14] Brice Loustau. The symplectic geometry of the deformation space of complex projective structures on a surface. $\operatorname{arXiv:1406.1821,} 2014$.

[McM00] Curtis T. McMullen. The moduli space of Riemann surfaces is Kähler hyperbolic. Ann. of Math. (2), 151(1):327-357, 2000.

[RS99] Igor Rivin and Jean-Marc Schlenker. The Schläfli formula in Einstein manifolds with boundary. Electron. Res. Announc. Amer. Math. Soc., 5:18-23 (electronic), 1999.

[Tau04] Clifford Henry Taubes. Minimal surfaces in germs of hyperbolic 3manifolds. In Proceedings of the Casson Fest, volume 7 of Geom. Topol. Monogr., pages 69-100 (electronic). Geom. Topol. Publ., Coventry, 2004.

[Thu97] William P. Thurston. Three-dimensional geometry and topology. Vol. 1, volume 35 of Princeton Mathematical Series. Princeton University Press, Princeton, NJ, 1997. Edited by Silvio Levy.

[Uhl83] Karen K. Uhlenbeck. Closed minimal surfaces in hyperbolic 3-manifolds. In Seminar on minimal submanifolds, volume 103 of Ann. of Math. Stud., pages 147-168. Princeton Univ. Press, Princeton, NJ, 1983. 